\title{
Variations in the Peptidase Activities of Escherichia coli in Response to Environmental Changes
}

\author{
By J. W. PAY NE \\ Microbiological Research Establishment, Porton, Salisbury, Wiltshire
}

(Accepted for publication 27 January 1972)

\begin{abstract}
SUMMARY
The activities of soluble, intracellular $\mathrm{Co}^{2+}$ and $\mathrm{Mn}^{2+}$ activated dipeptidases of Escherichia coli varied with the growth conditions. With chemostat cultures, potassium-limitation and oxygen depletion produced high peptidase activities whereas activities were low in carbon-limited cultures; the activity of carbon-limited organisms approximated to that of exponential-phase batch-grown organisms. The effect of growth conditions on the specific activity of soluble tripeptidase, which did not require cations for activity, was different from that found for the dipeptidases; the highest activity was again found in potassium-limited organisms but lowest activity occurred with nitrogen and magnesium limitation. The activities of all these peptidases were independent of the $\mathrm{pH}$ of growth, within the range $\mathrm{pH}$ $7 \cdot 0$ to $5 \cdot 5$. These results are discussed in relation to the utilization of peptides by growing organisms and to measurements of the turnover of protein during growth.
\end{abstract}

\section{INTRODUCTION}

Although intracellular peptidases are required to carry out certain specific metabolic functions, e.g. deformylation of polypeptide chains during protein maturation, their role during the bacterial growth cycle is unclear (Sussman \& Gilvarg, 197I), and indeed it has been suggested that they may exist in a latent form (Simmonds, 1970). However, active peptidases do occur within Escherichia coli. For example, amino acid auxotrophs grow equally well whether their required amino acids are supplied in peptide form, or as the free amino acids (Payne \& Gilvarg, I97I). Furthermore, although early reports suggested that protein turnover does not occur in exponentially growing organisms (Rotman \& Spiegelman, I954; Hogness, Cohn \& Monod, I955; Koch \& Levy, I955; Mandelstam, 1960), it is now clear that a small pool of labile proteins is degraded to amino acids during exponential growth (Willetts, I967 $b$; Nath \& Koch, 1970; Sussman \& Gilvarg, 1970). A fraction of the total protein in E. coli may be degraded at the time of bacterial division while proteolysis does not occur at other times; these suggestions have been correlated with rhythmic variations in peptidase activities during the growth cycle with maximum activities being exhibited at the fission periods (Kogoma \& Nishi, I965; Nishi \& Kogoma, 1965; Nishi \& Hirose, I966).

We have observed that the auxotrophic growth response of Escherichia coli to certain peptides varied with the type of inocula used (Payne, I97I; Payne \& Gilvarg, 1971), and similar results have been reported by other workers (Stone, I953; Simmonds, 1970). In certain cases, with exponential-phase batch-grown organisms as inocula, a prolonged lag period may precede exponential growth, whereas if the inoculum comprises stationary-phase organisms immediate exponential growth is observed (Stone, I953; Payne, 1971), while the exact opposite has been observed with other strains using different peptides (Simmonds, 
1970). These observations seem to correlate with variability of intracellular peptidase activities with growth phase (Simmonds, I970). However, in attempts to elucidate a mechanism for the control of peptidase activities, it is often difficult with batch grown organisms to find why growth stops which, coupled with the unknown effects of changes in the culture medium during the growth cycle, has prompted us to study the problem using organisms grown in the controlled environment of the chemostat. In this paper we describe the variations in the peptidase activity of $E$. coli that occur in response to specific nutrient limitations and to variations in the culture $\mathrm{pH}$; the results are related to the nutritional utilization of peptides and to the role of peptidases in protein turnover.

\section{METHODS}

Chemicals. Peptides were purchased from the sources described elsewhere (Payne, 1972); $o$-nitrophenyl- $\beta$-D-galactopyranoside was obtained from Sigma (London) Chemical Co. Ltd, London, S.W.6.

Growth of bacteria and preparation of crude extracts. Escherichia coli B (MRE strain 160 ) was grown in a conventional Porton-type chemostat of $500 \mathrm{ml}$ working capacity. Temperature was controlled at $37^{\circ} \mathrm{C}$, the $\mathrm{pH}$ was kept constant by automatic titration with $2 \mathrm{M}-\mathrm{NaOH}$, and the culture atmosphere was supplied at the rate of one reactor volume/min. Growth media was pumped continuously to give a dilution rate (proportion of the reactor volume flowing/h) of about $0 \cdot 2$. The defined media used were the series devised in this laboratory (Evans, Herbert \& Tempest, 1970), and the concentration of the limiting substrates was at a level which gave a yield of organisms up to $4.5 \mathrm{mg} / \mathrm{ml}$, dry wt. For anaerobic growth, oxygen-free nitrogen was used with the same carbon-limited medium as for aerobic growth. However, since the carbon source is also required to provide energy, and more energy is needed in anaerobiosis, utilization of the glucose for growth was reduced, and the yield fell to about $\mathrm{x} \cdot 3 \mathrm{mg}$ dry wt bacteria/ml. For growth at different $\mathrm{pH}$ values, the culture was first equilibrated at the required $\mathrm{pH}$ under carbon-limited conditions (the concentration of glucose was $20 \%$ of that used for normal carbon limitation, dry wt yield $0.85 \mathrm{mg} / \mathrm{ml}$ ). A 'burst' addition of glucose was then made which allowed the organisms to grow exponentially without limitation until this excess glucose was exhausted; bacteria were collected during this unlimited growth period. Effluents from the reactor were then taken into icecooled receivers, the organisms collected by centrifuging and either disrupted immediately or stored as wet pastes at $-20^{\circ} \mathrm{C}$. For batch culture, organisms were grown in 31 flasks in unlimited medium ( $400 \mathrm{ml}$ ) (Evans et al. 1970), with vigorous shaking at $37^{\circ} \mathrm{C}$, and were harvested in exponential phase. The yields of organisms grown under these different cultural conditions are shown in Table I. To prepare bacteria-free extracts, organisms were washed once in Io mM-potassium phosphate buffer, $\mathrm{pH} 7 \cdot \mathrm{I}$, and after centrifuging were resuspended in the same buffer at approximately $5 \times 10^{10}$ organisms $/ \mathrm{ml}$ for disruption by either of two procedures. For larger volumes, a Braun cell homogenizer MSK (Shandon Scientific Co. Ltd, North Circular Road, London, N.W.10) was used. The bacterial suspension was mixed with an equal volume of grade 12 ballotini beads, and the organisms were disrupted by shaking for Io min with $\mathrm{CO}_{2}$ cooling, using the standard procedure suggested by the manufacturer. The suspension of broken organisms was filtered through a glass sinter to remove beads, and the filtrate was separated from debris by centrifuging at I $5000 \mathrm{~g}$ for $\mathrm{I} \mathrm{h}$. Smaller volumes of organisms were disrupted by ultrasound using an MSE probe and Mullard power unit; the probe was tuned to optimum efficiency by ear. The suspended bacteria were cooled in ice, sonicated for I5 min, and finally centrifuged as above. The crude extracts were stored at $-20{ }^{\circ} \mathrm{C}$. 
Table I. Growth of Escherichia coli $\mathrm{B}$ under different nutrient conditions

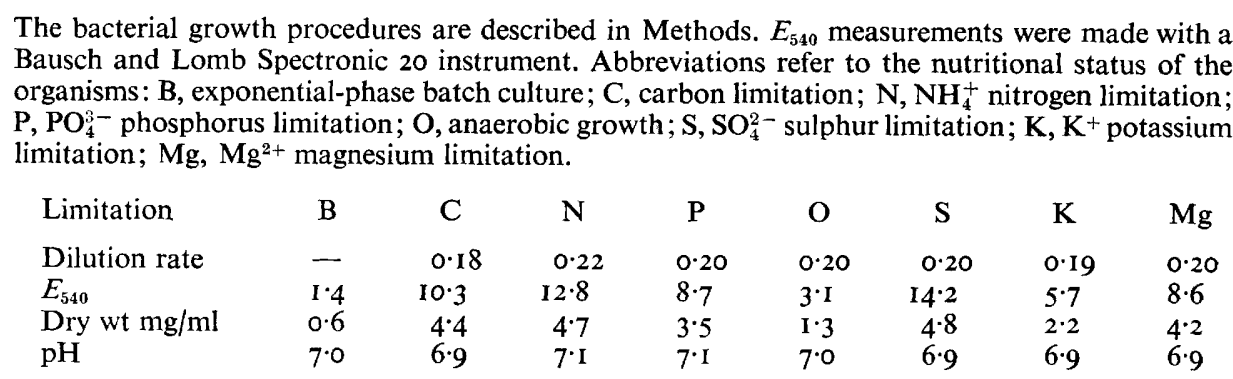

Assays. Peptidase assays were performed as described elsewhere (Payne, 1972). $\beta$ Galactosidase activity was measured by the procedure of Hestrin, Feingold \& Schramm (I955).

\section{RESULTS}

Earlier work on the characterization of the soluble peptidases of Escherichia coli established optimum conditions for their assay in extracts (Payne, 1972); these conditions have been employed here in studies of the influence of culture conditions on the activities of intracellular peptidases. Table 2 shows the hydrolytic activities towards diglycine of extracts of organisms grown under different nutrient limitations. The unsupplemented extracts displayed uniformly low activities, but hydrolysis was markedly stimulated in all cases by the addition of either $\mathrm{Co}^{2+}$ or $\mathrm{Mn}^{2+}$. Cleavage was stimulated to a greater extent with $\mathrm{Co}^{2+}$ than with $\mathrm{Mn}^{2-}$, and with $\mathrm{Co}^{2+}$ maximum activity was obtained using phosphate buffer whereas with $\mathrm{Mn}^{2}$ almost equal activities were observed in phosphate and in tris. The activating effect of both $\mathrm{Co}^{2+}$ and $\mathrm{Mn}^{2+}$ was counteracted by $\mathrm{Zn}^{2+}$. In general, EDTA was without marked effect on the $\mathrm{Co}^{2+}$ activated hydrolysis but with all extracts it enhanced activity in the presence of $\mathrm{Mn}^{2+}$. In both buffers, the different extracts displayed a three- to fourfold variation in their rates of cleavage of diglycine in the presence of $\mathrm{Co}^{2+}$, and a similar distribution of activities was seen for $\mathrm{Mn}^{2+}$ activation. In both cases, greatest activity was found for organisms grown under potassium limitation or anaerobically.

With prolylglycine (Table 3 ), the overall pattern of peptidase activity was similar to that with diglycine: low activity with unsupplemented extracts, and $\mathrm{Co}^{2+}$ enhancing activity to a greater extent than $\mathrm{Mn}^{2+}$, and only with $\mathrm{Co}^{2+}$ did phosphate stimulate activity. Activity with $\mathrm{Mn}^{2+}$ (but not with $\mathrm{Co}^{2+}$ ) was again enhanced by EDTA; $\mathrm{Zn}^{2+}$ inhibited activity with $\mathrm{Co}^{2+}$ or $\mathrm{Mn}^{2+}$. The $\mathrm{Co}^{2+}$ peptidase was again present in the greatest activity in potassium-limited and anaerobically-grown bacteria and the activities of other extracts fell between these values and those of the lowest activities found with batch-grown and carbon-limited organisms. The $\mathrm{Mn}^{2+}$ stimulated activity was distributed in a similar manner to the $\mathrm{Co}^{2+}$ activated enzyme, with batch-grown and carbon-limited organisms possessing only about $15 \%$ of the maximum activity observed with potassium-limited organisms.

Results for the cleavage of divaline are shown in Table 4 . The greatest activity $\left(\mathrm{Co}^{2+}\right.$ stimulated) was again found in potassium-limited organisms; activities of bacteria grown under other limitations were very similar, the least active being batch-grown and carbonlimited organisms. With divaline as substrate there was little difference in the activities measured in phosphate and in tris buffers. Activity was inhibited by $\mathrm{Zn}^{2+}$, more so in tris than in phosphate. With the $\mathrm{Mn}^{2+}$ activated enzyme, activity was once again greatest with 
Table 2. Cleavage of diglycine by extracts of Escherichia coli grown under various nutrient conditions

Incubation mixtures $(\mathrm{I} \mathrm{ml})$ contained diglycine $(7.5 \mathrm{~mm})$ and either potassium phosphate $\mathrm{pH} 7.5$ $(20 \mathrm{mM}), \mathrm{P}$, or tris- $\mathrm{HCl} \mathrm{pH} \mathrm{7.9}(20 \mathrm{mM}), \mathrm{T}$. The following were added as indicated: $\mathrm{Co}^{2+}(\mathrm{I} \cdot 0 \mathrm{~mm})$; $\mathrm{Mn}^{2+}(\mathrm{I} \cdot 0 \mathrm{~mm}) ;$ EDTA $(0.25 \mathrm{~mm}) ; \mathrm{Zn}^{2+}(0.5 \mathrm{~mm})$. Crude extract was added to give approximately $500 \mu \mathrm{g}$ protein $/ \mathrm{ml}$ with buffers alone, approximately $20 \mu \mathrm{g}$ protein $/ \mathrm{ml}$ with added $\mathrm{Co}^{2+}$ and about $75 \mu \mathrm{g}$ protein $/ \mathrm{ml}$ with $\mathrm{Mn}^{2+}$ supplemented mixtures. Peptidase assays were performed as described in Methods. Peptidase activity is expressed as $\mu \mathrm{mol}$ glycine released $/ \mathrm{min} / \mathrm{mg}$ protein. Abbreviations indicating nutrient status of organisms are shown in legend to Table 1 ; n.d. = not determined, $\mathbf{P}=$ phosphate, $\mathbf{T}=$ tris.

Crude extract supplemented with

$\mathbf{P}$

T

$\mathrm{P}+\mathrm{Co}^{2+}$

$\mathrm{P}+\mathrm{Co}^{2+}+\mathrm{EDTA}$

$\mathrm{P}+\mathrm{Co}^{2+}+\mathrm{Zn}^{2+}$

$\mathrm{T}+\mathrm{Co}^{2+}$

$\mathrm{T}+\mathrm{Co}^{2+}+$ EDTA

$\mathrm{T}+\mathrm{Co}^{2+}+\mathrm{Zn}^{2+}$

$\mathrm{P}+\mathrm{Mn}^{2+}$

$\mathrm{P}+\mathrm{Mn}^{2+}+$ EDTA

$\mathrm{P}+\mathrm{Mn}^{2+}+\mathrm{Zn}^{2+}$

$\mathrm{T}+\mathrm{Mn}^{2+}$

$\mathrm{T}+\mathrm{Mn}^{2+}+$ EDTA

$\mathrm{T}+\mathrm{Mn}^{2+}+\mathrm{Zn}^{2+}$

Peptidase activity ( $\mu \mathrm{mol} / \mathrm{min} / \mathrm{mg}$ protein)

\begin{tabular}{|c|c|c|c|c|c|c|c|}
\hline B & $\mathrm{C}$ & $\mathrm{N}$ & $\mathbf{P}$ & $\mathrm{O}$ & $S$ & $\mathrm{~K}$ & $\mathrm{Mg}$ \\
\hline 0.07 & 0.01 & 0.04 & 0.07 & 0.01 & 0.02 & 0.08 & 0.06 \\
\hline 0.03 & 0.02 & 0.04 & 0.12 & 0.01 & 0.02 & 0.11 & 0.06 \\
\hline $7 \cdot 60$ & $4 \cdot 84$ & $7 \cdot 4^{2}$ & 9.90 & I I $\cdot 25$ & $8 \cdot 10$ & I 4.90 & 6.83 \\
\hline $8 \cdot 05$ & 5.25 & $7 \cdot 42$ & $9 \cdot 90$ & $12 \cdot 10$ & $8 \cdot 10$ & 14.90 & $7 \cdot 77$ \\
\hline n.d. & 0.78 & 0.11 & 0.94 & n.d. & n.d. & n.d. & n.d. \\
\hline $1 \cdot 50$ & 0.91 & $I \cdot I 8$ & $I \cdot 72$ & $4 \cdot 24$ & $2 \cdot 68$ & $6 \cdot 88$ & I. 68 \\
\hline$I \cdot I O$ & 0.55 & 0.78 & $\mathrm{I} \cdot 4 \mathrm{I}$ & 3.67 & $2 \cdot 68$ & $7 \cdot 65$ & 0.95 \\
\hline n.d. & 0.03 & 0.00 & 0.00 & n.d. & n.d. & n.d. & n.d. \\
\hline 0.66 & 0.85 & $I \cdot 00$ & 0.57 & $I \cdot 13$ & $I \cdot 52$ & $2 \cdot 75$ & I. 32 \\
\hline $1 \cdot 00$ & $I \cdot 00$ & $1 \cdot 49$ & 0.83 & 2.52 & $2 \cdot 08$ & 4.05 & I. 49 \\
\hline n.d. & 0.03 & 0.02 & 0.06 & n.d. & n.d. & n.d. & n.d. \\
\hline 0.69 & 0.84 & 0.82 & $0.8 \mathrm{I}$ & $1 \cdot 48$ & I 54 & $3 \cdot 34$ & $I \cdot 32$ \\
\hline $1 \cdot 00$ & 1.03 & $1 \cdot 35$ & 0.76 & 2.52 & $1 \cdot 38$ & 3.50 & $1 \cdot 60$ \\
\hline n.d. & 0.03 & 0.02 & 0.00 & n.d. & n.d. & n.d. & n.d. \\
\hline
\end{tabular}

Table 3. Cleavage of prolylglycine by extracts of Escherichia coli grown under various nutrient limitations

Incubation mixtures ( $\mathrm{I} \mathrm{ml}$ ) contained prolylglycine $(7.5 \mathrm{~mm})$ and either potassium phosphate pH 7.5 (20 mM) or tris- $\mathrm{HCl} \mathrm{pH} \mathrm{7.9}(20 \mathrm{mM})$. The following were added as indicated: $\mathrm{Co}^{2}+(\mathrm{I} \cdot \mathrm{O} \mathrm{mM})$; $\mathrm{Mn}^{2+}(\mathrm{I} \cdot \mathrm{OmM})$; EDTA $(0.25 \mathrm{~mm}) ; \mathrm{Zn}^{2+}(0.5 \mathrm{~mm})$. Crude extract was added to give approximately $1000 \mu \mathrm{g}$ protein $/ \mathrm{ml}$ with buffers alone, approximately $150 \mu \mathrm{g}$ protein $/ \mathrm{ml}$ with $\mathrm{Co}^{2+}$ and about $750 \mu \mathrm{g}$ protein/ml with $\mathrm{Mn}^{2+}$ supplemented mixtures. Peptidase assays were performed as described in Methods. Peptidase activity is expressed as $\mu \mathrm{mol}$ amino acid released $/ \mathrm{min} / \mathrm{mg}$ protein. Abbreviations indicating nutrient status of organisms are shown in legend to Table $\mathrm{I}$; n.d. $=$ not determined, $\mathrm{P}=$ phosphate, $\mathrm{T}=$ tris.

Peptidase activity $(\mu \mathrm{mol} / \mathrm{min} / \mathrm{mg}$ protein)

\begin{tabular}{|c|c|c|c|c|c|c|c|c|}
\hline $\begin{array}{c}\text { Crude extract } \\
\text { supplemented with }\end{array}$ & B & $\mathrm{C}$ & $\mathbf{N}$ & $\mathbf{P}$ & $\mathrm{O}$ & S & $\mathbf{K}$ & $\mathrm{Mg}$ \\
\hline $\mathbf{P}$ & 0.02 & $0.0 \mathrm{I}$ & 0.00 & 0.02 & $0.0 \mathrm{I}$ & 0.01 & $0.0 \mathrm{I}$ & $0.0 \mathrm{I}$ \\
\hline $\mathbf{T}$ & 0.01 & 0.00 & 0.03 & $0.0 \mathrm{I}$ & 0.00 & 0.01 & O.OI & 0.01 \\
\hline $\mathrm{P}+\mathrm{Co}^{2+}$ & 0.82 & 0.54 & 1.09 & $I \cdot 35$ & $\mathrm{I} \cdot 32$ & $\mathrm{I} \cdot 2 \mathrm{I}$ & $2 \cdot 35$ & $\mathrm{I} \cdot 03$ \\
\hline $\mathrm{P}+\mathrm{Co}^{2+}+\mathrm{EDTA}$ & 0.82 & 0.54 & $\mathrm{I} \cdot 09$ & $I \cdot 35$ & $I \cdot 32$ & $\mathrm{I} \cdot 2 \mathrm{I}$ & $2 \cdot 35$ & $\mathrm{I} \cdot 03$ \\
\hline $\mathrm{P}+\mathrm{Co}^{2+}+\mathrm{Zn}^{2+}$ & n.d. & 0.04 & 0.09 & 0.14 & n.d. & n.d. & n.d. & n.d. \\
\hline $\mathrm{T}+\mathrm{Co}^{2+}$ & 0.49 & 0.36 & 0.56 & 0.56 & $1 \cdot 00$ & 0.65 & 0.92 & 0.67 \\
\hline $\mathrm{T}+\mathrm{Co}^{2+}+\mathrm{EDTA}$ & 0.38 & 0.36 & 0.56 & 0.56 & 0.77 & 0.65 & 0.92 & 0.67 \\
\hline $\mathrm{T}+\mathrm{Co}^{2+}+\mathrm{Zn}^{2+}$ & n.d. & 0.03 & 0.00 & 0.00 & n.d. & n.d. & n.d. & n.d. \\
\hline $\mathrm{P}+\mathrm{Mn}^{2+}$ & 0.04 & 0.12 & 0.07 & 0.12 & 0.13 & 0.12 & 0.36 & 0.17 \\
\hline $\mathrm{P}+\mathrm{Mn}^{2+}+$ EDTA & $0.1 \mathrm{I}$ & 0.13 & 0.17 & 0.25 & 0.25 & 0.25 & 0.83 & 0.20 \\
\hline $\mathrm{P}+\mathrm{Mn}^{2+}+\mathrm{Zn}^{2+}$ & n.d. & 0.03 & 0.00 & 0.01 & n.d. & n.d. & n.d. & n.d. \\
\hline $\mathrm{T}+\mathrm{Mn}^{2+}$ & 0.04 & 0.12 & 0.18 & $0 \cdot 20$ & 0.19 & 0.32 & 0.39 & 0.17 \\
\hline $\mathrm{T}+\mathrm{Mn}^{2+}+\mathrm{EDTA}$ & 0.1 I & 0.15 & $0.3 \mathrm{I}$ & 0.14 & 0.26 & 0.32 & 0.96 & 0.29 \\
\hline $\mathrm{T}+\mathrm{Mn}^{2+}+\mathrm{Zn}^{2+}$ & n.d. & 0.04 & 0.00 & 0.02 & n.d. & n.d. & n.d. & n.d \\
\hline
\end{tabular}


Table 4. Cleavage of divaline by extracts of Escherichia coli grown under various nutrient conditions

Incubation mixtures ( $\mathrm{I} \mathrm{ml})$ contained divaline $(7.5 \mathrm{~mm})$, and either potassium phosphate $\mathrm{pH} 7.5$ $(20 \mathrm{mM})$ or tris- $\mathrm{HCl} \mathrm{pH} 7.9(20 \mathrm{mM})$. The following were added as indicated: $\mathrm{Co}^{2+}(\mathrm{I} \cdot \mathrm{O} \mathrm{mM})$; $\mathrm{Mn}^{2+}(\mathrm{I} \cdot 0 \mathrm{mM}) ;$ EDTA $(0.25 \mathrm{~mm}) ; \mathrm{Zn}^{2+}(0.5 \mathrm{mM})$. Crude extract was added to give approximately $1000 \mu \mathrm{g}$ protein $/ \mathrm{ml}$ with buffers alone, approximately $250 \mu \mathrm{g}$ protein $/ \mathrm{ml}$ with added $\mathrm{Co}^{2+}$ and about I $000 \mu \mathrm{g}$ protein $/ \mathrm{ml}$ with $\mathrm{Mn}^{2+}$ supplemented mixtures. Peptidase assays were performed as described in Methods. Peptidase activity is expressed as $\mu \mathrm{mol}$ valine released $/ \mathrm{min} / \mathrm{mg}$ protein. Abbreviations indicating nutrient status of organisms are shown in legend to Table $\mathrm{I} ;$ n.d. = not determined, $\mathbf{P}=$ phosphate, $\mathbf{T}=$ tris.

Crude extract supplemented with

$\mathbf{P}$

$\mathrm{T}$

$\mathbf{P}+\mathrm{Co}^{2+}$

$\mathrm{P}+\mathrm{Co}^{2+}+\mathrm{EDTA}$

$\mathrm{P}+\mathrm{Co}^{2+}+\mathrm{Zn}^{2+}$

$\mathrm{T}+\mathrm{Co}^{2+}$

$\mathrm{T}+\mathrm{Co}^{2+}+$ EDTA

$\mathrm{T}+\mathrm{Co}^{2+}+\mathrm{Zn}^{2+}$

$\mathrm{P}+\mathrm{Mn}^{2+}$

$\mathrm{P}+\mathrm{Mn}^{2+}+$ EDTA

$\mathrm{P}+\mathrm{Mn}^{2+}+\mathrm{Zn}^{2+}$

$\mathrm{T}+\mathrm{Mn}^{2+}$

$\mathrm{T}+\mathrm{Mn}^{2+}+$ EDTA

$\mathrm{T}+\mathrm{Mn}^{2+}+\mathrm{Zn}^{2+}$

Peptidase activity ( $\mu \mathrm{mol} / \mathrm{min} / \mathrm{mg}$ protein)

\begin{tabular}{cccccccc}
\hline B & C & N & P & O & S & K & Mg \\
0.02 & 0.01 & 0.01 & 0.03 & 0.01 & 0.03 & 0.01 & 0.05 \\
0.01 & 0.00 & 0.02 & 0.03 & 0.00 & 0.03 & 0.00 & 0.05 \\
0.24 & 0.41 & 0.56 & 0.59 & 0.53 & 0.59 & 0.81 & 0.59 \\
0.24 & 0.38 & 0.56 & 0.59 & 0.53 & 0.59 & 0.81 & 0.59 \\
n.d. & 0.03 & 0.24 & n.d. & n.d. & n.d. & n.d. & n.d. \\
0.18 & 0.22 & 0.48 & 0.56 & 0.62 & 0.56 & 0.82 & 0.55 \\
0.18 & 0.27 & 0.48 & 0.46 & 0.62 & 0.46 & 0.82 & 0.44 \\
n.d. & 0.01 & 0.06 & n.d. & n.d. & n.d. & n.d. & n.d. \\
0.06 & 0.04 & 0.12 & 0.07 & 0.13 & 0.07 & 0.18 & 0.10 \\
0.06 & 0.04 & 0.11 & 0.07 & 0.13 & 0.07 & 0.15 & 0.08 \\
n.d. & 0.02 & 0.04 & n.d. & n.d. & n.d. & n.d. & n.d. \\
0.02 & 0.04 & 0.09 & 0.10 & 0.09 & 0.10 & 0.06 & 0.11 \\
0.04 & 0.05 & 0.05 & 0.10 & 0.11 & 0.10 & 0.06 & 0.11 \\
n.d. & 0.01 & 0.03 & n.d. & n.d. & n.d. & n.d. & n.d.
\end{tabular}

\section{Table 5. Cleavage of triglycine by extracts of Escherichia coli} grown under various nutrient conditions

Incubation mixtures ( $\mathrm{I} \mathrm{ml}$ ) contained triglycine $(7.5 \mathrm{~mm}$ ) and either potassium phosphate $\mathrm{pH} 7.5$ $(20 \mathrm{~mm})$ or tris- $\mathrm{HCl} \mathrm{pH} \mathrm{7.9}(20 \mathrm{~mm})$. The following were added as indicated: $\mathrm{Co}^{2+}(\mathrm{I} \cdot \mathrm{O} \mathrm{mm})$; $\mathrm{Mn}^{2+}(\mathrm{I} \cdot 0 \mathrm{mM}) ;$ EDTA $(0.25 \mathrm{mM}) ; \mathrm{Zn}^{2+}(0.5 \mathrm{mM})$. Crude extract was added to give approximately I000 $\mu \mathrm{g}$ protein $/ \mathrm{ml}$ with buffers alone, approximately $150 \mu \mathrm{g}$ protein $/ \mathrm{ml}$ with added $\mathrm{Co}^{2+}$ and about $750 \mu \mathrm{g}$ protein $/ \mathrm{ml}$ with $\mathrm{Mn}^{2+}$ supplemented mixtures. Peptidase assays were performed as described in Methods. Peptidase activity is expressed as $\mu \mathrm{mol}$ glycine released $/ \mathrm{min} / \mathrm{mg}$ protein. Abbreviations indicating nutrient status of organisms are shown in legend to Table $\mathrm{I}$; n.d. = not determined, $\mathrm{P}=$ phosphate, $\mathrm{T}=$ tris.

\begin{tabular}{|c|c|c|c|c|c|c|c|c|}
\hline \multirow{2}{*}{$\begin{array}{c}\text { Crude extract } \\
\text { supplemented with }\end{array}$} & \multicolumn{8}{|c|}{ s. } \\
\hline & B & $\mathrm{C}$ & $\mathbf{N}$ & $\mathbf{P}$ & $\mathrm{O}$ & $\mathbf{S}$ & $\mathbf{K}$ & $\mathrm{Mg}$ \\
\hline $\mathbf{P}$ & 0.09 & 0.06 & 0.09 & 0.09 & 0.04 & 0.14 & 0.15 & 0.09 \\
\hline $\mathbf{T}$ & 0.07 & 0.08 & $0.1 \mathrm{I}$ & 0.12 & 0.13 & $0 \cdot 19$ & $0 \cdot 19$ & $0.1 \mathrm{I}$ \\
\hline $\mathrm{P}+\mathrm{Co}^{2+}$ & 0.93 & 0.99 & 0.64 & 0.88 & 0.85 & $1 \cdot 15$ & $\mathrm{I} \cdot 20$ & 0.64 \\
\hline $\mathrm{P}+\mathrm{Co}^{2+}+\mathrm{EDTA}$ & 0.83 & 0.96 & 0.75 & 0.88 & 0.91 & I. I 5 & $1 \cdot 38$ & 0.75 \\
\hline $\mathrm{P}+\mathrm{Co}^{2+}+\mathrm{Zn}^{2+}$ & n.d. & n.d. & 0.13 & 0.37 & n.d. & n.d. & n.d. & 0.13 \\
\hline $\mathrm{T}+\mathrm{Co}^{2+}$ & 0.46 & 0.83 & 0.14 & 0.80 & 0.52 & $1 \cdot 06$ & $\mathrm{I} \cdot \mathrm{I} 7$ & $\mathrm{O} \cdot \mathrm{I} 4$ \\
\hline $\mathrm{T}+\mathrm{Co}^{2+}+\mathrm{EDTA}$ & 0.46 & 0.69 & 0.23 & 0.72 & 0.69 & $I \cdot 00$ & $1 \cdot 17$ & 0.23 \\
\hline $\mathrm{T}+\mathrm{Co}^{2+}+\mathrm{Zn}^{2+}$ & n.d. & n.d. & 0.02 & 0.09 & n.d. & n.d. & n.d. & 0.02 \\
\hline $\mathrm{P}+\mathrm{Mn}^{2+}$ & 0.16 & 0.09 & 0.13 & 0.19 & 0.14 & 0.23 & 0.29 & 0.13 \\
\hline $\mathrm{P}+\mathrm{Mn}^{2+}+\mathrm{EDTA}$ & 0.16 & 0.17 & 0.18 & 0.22 & 0.21 & 0.27 & 0.30 & 0.18 \\
\hline $\mathbf{P}+\mathbf{M n}^{2+}+\mathbf{Z n}^{2+}$ & n.d. & n.d. & 0.08 & 0.09 & n.d. & n.d. & n.d. & 0.08 \\
\hline $\mathrm{T}+\mathrm{Mn}^{2+}$ & 0.14 & 0.15 & $0.2 \mathrm{I}$ & 0.23 & $0 \cdot 19$ & 0.31 & 0.31 & 0.21 \\
\hline $\mathrm{T}+\mathrm{Mn}^{2+}+\mathrm{EDTA}$ & 0.19 & 0.17 & $0 \cdot 21$ & 0.21 & 0.21 & $0 \cdot 31$ & 0.29 & $0.2 \mathrm{I}$ \\
\hline $\mathrm{T}+\mathrm{Mn}^{2+}+\mathrm{Zn}^{2+}$ & n.d. & n.d. & 0.09 & 0.05 & n.d. & n.d. & n.d. & 0.09 \\
\hline
\end{tabular}



Table 6. The peptidase activity of extracts of Escherichia coli grown
at various $\mathrm{pH}$ values

Incubation mixtures $(1.0 \mathrm{ml})$ contained peptide $(7.5 \mathrm{~mm})$ and either potassium phosphate $\mathrm{pH} 7.5$ $(20 \mathrm{mM})$ or tris- $\mathrm{HCl} \mathrm{pH} \mathrm{7.9}(20 \mathrm{mM})$. The following additions were made as indicated, $\mathrm{Co}^{2+}$ ( $.0 \mathrm{mM}) ; \mathrm{Mn}^{2+}$ (I.O mM); EDTA (0.25 mM). With diglycine, prolylglycine, divaline, triglycine and trivaline, crude extract was added to give approximately $100 \mu \mathrm{g}$ protein $/ \mathrm{ml}$ using $\mathrm{Co}^{2+}$ and $500 \mu \mathrm{g}$ protein/ml using $\mathrm{Mn}^{2+}$. Peptidase assays were performed as described in Methods. Peptidase activity is expressed as $\mu \mathrm{mol}$ amino acid released $/ \mathrm{min} / \mathrm{mg}$ protein. $\mathrm{n} . \mathrm{d} .=$ Not determined, $\mathrm{P}=$ phosphate, $\mathrm{T}=$ tris.

Peptidase activity in extracts supplemented with

\begin{tabular}{|c|c|c|c|c|c|c|c|c|c|c|c|}
\hline Peptide & $\mathrm{pH}$ & $\mathbf{P}$ & $\mathrm{T}$ & $\begin{array}{c}\mathbf{P} \\
\mathrm{Co}^{2+}\end{array}$ & $\begin{array}{c}\mathbf{P} \\
\mathrm{Co}^{2+} \\
\text { EDTA }\end{array}$ & $\begin{array}{c}\mathrm{T} \\
\mathrm{Co}^{2+}\end{array}$ & $\begin{array}{c}\mathrm{T} \\
\mathrm{Co}^{2+} \\
\text { EDTA }\end{array}$ & $\underset{\mathrm{Mn}^{2+}}{\mathbf{P}}$ & $\begin{array}{c}\mathbf{P} \\
\mathrm{Mn}^{2+} \\
\text { EDTA }\end{array}$ & $\underset{\mathrm{Mn}^{2+}}{\mathrm{T}}$ & $\begin{array}{c}\text { T } \\
\text { Mn }^{2+} \\
\text { EDTA }\end{array}$ \\
\hline Diglycine & $7 \cdot 0$ & $0.0 I$ & 0.01 & $7 \cdot 70$ & $7 \cdot 70$ & I. 00 & $I \cdot 00$ & 0.43 & 0.99 & 0.60 & $I \cdot 28$ \\
\hline Diglycine & $6 \cdot 5$ & 0.02 & 0.03 & $5 \cdot 80$ & $5 \cdot 80$ & I.65 & $1 \cdot 65$ & $0 \cdot 78$ & $I \cdot 25$ & 0.80 & $\mathrm{I} \cdot \mathrm{I} 5$ \\
\hline Diglycine & $6 \cdot 0$ & 0.06 & 0.07 & $5 \cdot 69$ & $5 \cdot 69$ & $I \cdot 60$ & $I \cdot 60$ & I'IO & I. 86 & $\mathrm{I} \cdot 57$ & $\mathrm{I} \cdot 57$ \\
\hline Diglycine & $5 \cdot 5$ & 0.01 & 0.04 & $6 \cdot 15$ & 6.15 & $I \cdot 68$ & $2 \cdot 25$ & $I \cdot I I$ & $I \cdot I I$ & $I \cdot I I$ & I'I I \\
\hline Prolylglycine & $7 \cdot 0$ & 0.01 & 0.01 & 0.86 & $0 \cdot 86$ & 0.63 & 0.63 & 0.15 & 0.18 & $0 \cdot 20$ & 0.26 \\
\hline Prolylglycine & $6 \cdot 5$ & 0.01 & 0.01 & $I \cdot I 6$ & $I \cdot 30$ & 0.72 & 0.72 & O.I I & OPI I & 0.28 & 0.28 \\
\hline Prolylglycine & $6 \cdot 0$ & 0.03 & 0.01 & $I \cdot 06$ & I.06 & 0.77 & 0.76 & $0 \cdot 26$ & 0.23 & 0.22 & 0.26 \\
\hline Prolylglycine & $5 \cdot 5$ & 0.01 & $0.0 I$ & $I \cdot I 3$ & $\mathrm{I} \cdot 38$ & 0.56 & 0.56 & 0.20 & 0.39 & 0.20 & 0.35 \\
\hline Divaline & $7 \cdot 0$ & $0.0 I$ & 0.01 & 0.27 & 0.27 & 0.27 & 0.27 & 0.05 & 0.05 & 0.05 & 0.05 \\
\hline Divaline & $6 \cdot 5$ & 0.03 & 0.01 & 0.30 & 0.30 & 0.26 & $0 \cdot 24$ & 0.05 & 0.05 & 0.05 & 0.05 \\
\hline Divaline & $6 \cdot 0$ & 0.07 & 0.01 & 0.33 & 0.33 & 0.35 & 0.42 & 0.08 & 0.04 & 0.05 & 0.06 \\
\hline Divaline & $5 \cdot 5$ & 0.04 & $0.0 I$ & 0.33 & 0.33 & 0.33 & 0.33 & 0.27 & 0.04 & 0.05 & 0.05 \\
\hline Triglycine & $7 \cdot 0$ & 0.12 & 0.05 & 0.66 & 0.66 & 0.39 & 0.39 & $0 \cdot 13$ & $0 \cdot 13$ & $0 \cdot 12$ & 0.12 \\
\hline Triglycine & $6 \cdot 5$ & 0.12 & $0 \cdot 13$ & 0.64 & 0.59 & $0 \cdot 38$ & 0.33 & 0.17 & $0 \cdot 16$ & $0 \cdot 19$ & 0.20 \\
\hline Triglycine & $6 \cdot 0$ & 0.08 & $0 \cdot 10$ & 0.68 & 0.73 & 0.41 & $0.4 I$ & 0.17 & 0.22 & $0.2 I$ & $0 \cdot 20$ \\
\hline Triglycine & $5 \cdot 5$ & 0.09 & 0.12 & $1 \cdot 44$ & $I \cdot 7 I$ & 0.90 & 0.60 & 0.29 & $0 \cdot 30$ & 0.31 & 0.29 \\
\hline Trivaline & $7 \cdot 0$ & 0.01 & 0.01 & $0 \cdot 13$ & 0.13 & 0.08 & 0.12 & n.d. & n.d. & n.d. & n.d. \\
\hline Trivaline & $6 \cdot 5$ & 0.02 & 0.02 & 0.14 & 0.17 & 0.04 & 0.05 & n.d. & n.d. & n.d. & n.d. \\
\hline Trivaline & 6.0 & 0.01 & 0.01 & 0.17 & 0.17 & $0 \cdot 10$ & 0.10 & n.d. & n.d. & n.d. & n.d. \\
\hline Trivaline & $5 \cdot 5$ & O.OI & $0.0 \mathrm{I}$ & 0.14 & $0 \cdot 14$ & 0.07 & 0.09 & n.d. & n.d. & n.d. & n.d. \\
\hline
\end{tabular}

potassium-limited organisms and least with carbon-limited, and batch-grown organism. With divaline, EDTA did not enhance activity, in contrast to the observations with diglycine and with prolylglycine.

With triglycine as substrate (Table 5) data are expressed in terms of glycine released and therefore in interpreting the results allowance should be made for amino acid released through dipeptidase action. The results differ somewhat from those observed with the above dipeptides, e.g. the absolute activities towards triglycine of the unsupplemented extracts were generally higher than towards dipeptides and the effects of supplements were less. With $\mathrm{Co}^{2+}$, the activities were uniformly lower than those found for diglycine hydrolysis suggesting that triglycine cleavage is the rate-limiting step. There was little variation in activity amongst different extracts, unlike the finding with dipeptide hydrolysis; what little variation did exist indicated that organisms grown under nitrogen and magnesium limitations had least activity, in contrast to the dipeptidases which displayed least activity in batch-grown, and carbon-limited, organisms. The activating effect of phosphate buffer and $\mathrm{Co}^{2+}$ was not as general or as marked as with diglycine. The limited effect of EDTA, and inhibition by $\mathrm{Zn}^{2+}$ resembled the findings with diglycine hydrolysis. Notably there was little variation in the hydrolytic activities towards triglycine of the various extracts supplemented with $\mathrm{Mn}^{2+}$, a result similar to that above for $\mathrm{Co}^{2+}$ but in contrast to the case with the 


\title{
Table 7. The $\beta$-galactosidase activity of extracts of Escherichia coli grown under various nutrient conditions
}

\begin{abstract}
Incubation mixtures $(4.5 \mathrm{ml})$ contained $o$-nitrophenyl- $\beta$-D-galactopyranoside $(\mathrm{I} \cdot \mathrm{I} \mathrm{mM})$, sodium phosphate buffer $\mathrm{pH} 7 \cdot 25$ ( $150 \mathrm{~mm}$ ) and crude extracts containing about I $\mathrm{mg}$ protein. Assays were performed as described in Methods. Activity is expressed as $\mu \mathrm{mol} \sigma$-nitrophenol released $/ \mathrm{h} / \mathrm{mg}$ protein.
\end{abstract}

\begin{tabular}{|c|c|c|c|}
\hline \multicolumn{4}{|c|}{$\beta$-Galactosidase activity of extracts } \\
\hline $\mathrm{pH}$ of growth* & Activity & $\begin{array}{l}\text { Nutrient } \\
\text { limitation }\end{array}$ & Activity \\
\hline $\mathrm{pH} 7 \cdot 0$ & $0 \cdot 34$ & B & 0.25 \\
\hline pH 6.5 & 0.48 & $\mathrm{C}$ & $1 \cdot 10$ \\
\hline $\mathrm{pH} 6.0$ & 0.48 & $\mathrm{~N}$ & 0.05 \\
\hline \multirow[t]{5}{*}{$\mathrm{pH} 5.5$} & 0.38 & $\mathbf{P}$ & 0.10 \\
\hline & & $\mathrm{O}$ & $1 \cdot \infty$ \\
\hline & & $\mathbf{S}$ & $0 \cdot 19$ \\
\hline & & $\mathrm{K}$ & 0.42 \\
\hline & & $\mathrm{Mg}$ & $0 \cdot 13$ \\
\hline
\end{tabular}

dipeptidases; what little variation there was did not closely follow the distribution seen with the dipeptidases. In general, EDTA has little stimulatory effect on activity with $\mathrm{Mn}^{2+}$, contrasting with the observations on diglycine.

Table 6 shows the influence of $\mathrm{pH}$ of the growth medium upon the levels of peptidase activities. The rate of dipeptide hydrolysis for all three substrates was essentially independent of the $\mathrm{pH}$ at which the bacteria were grown. With triglycine the data suggest that tripeptidase activity may increase as the $\mathrm{pH}$ of growth is decreased, although this conclusion is not endorsed by the results with trivaline which was cleft at a similar rate by all extracts.

$\beta$-Galactosidase activity of the various extracts was strongly influenced by the conditions of growth (Table 7). Activity was least in nitrogen-limited organisms which contain only about $5 \%$ of the activity of carbon-limited organisms. The distribution of $\beta$-galactosidase activity in no way paralleled that for the peptidases, suggesting that protein denaturation to varying extents during preparation of the different extracts is not an explanation for the varied activities. For comparisons of enzymic activities such as those described here, it would be most useful to have several constitutive enzymes that remained essentially invariant with growth conditions and therefore could serve as 'internal markers' of such effects as differential protein denaturation. However, it is doubtful whether such invariant behaviour can ever occur in practice, and certainly $\beta$-galactosidase does not fulfil such a role (Mandelstam, 196I).

\section{DISCUSSION}

The nutrient status of the organism influences the activities of the intracellular peptidases of Escherichia coli. Although other studies of the influence of medium composition upon $E$. coli peptidases have been reported (Simmonds \& Toye, 1967; Simmonds, 1970), these employed batch-grown organisms and therefore lacked the strict environmental control possible with chemostat cultures. However, for comparison we also studied the peptidase content of exponential-phase batch-grown organisms. We have intentionally limited our studies to extracts, since the changes of wall composition and structure that may occur in 
response to different nutrient limitations (Ellwood \& Tempest, I969) might well affect peptide permeability and thus make peptidase assays of whole organisms unreliable. To some extent, the same objection applies to assays with bacteria treated with toluene, which may disrupt the permeability barriers with variable efficiency depending upon the nature of the cell envelope. On the other hand, it is assumed that during disruption the soluble peptidases are uniformly released, and that differential binding of peptidases to cellular debris, which may vary in degree as a function of growth conditions, does not occur.

Others have shown that Escherichia coli peptidases are cytoplasmic enzymes (Matheson \& Murayama, 1966; Simmonds \& Toye, 1966; Van Lenten \& Simmonds, 1967) that are found whether or not peptides are in the growth medium (Matheson \& Tsai, I965; Simmonds \& Toye, I967; Simmonds, 1970). Their constitutive nature suggests that peptidases may be required for physiological functions in addition to the nutritional one of cleavage of exogenous peptides (Sussman \& Gilvarg, 197I). In this context, one may calculate the peptidase activity that is needed to satisfy the amino acid requirement of an auxotroph when the amino acid is supplied in peptide form; this is shown in the appendix for divaline. Which experimental value should be compared to such a calculated activity is uncertain. It can be argued (Simmonds, 1970) that addition of cations to crude extracts activates peptidases that are latent within the bacterium, and that such activities are therefore an overestimate of intracellular activity in vivo. In contrast, during preparation of crude extracts, essential cofactors may be diluted away, so that addition of cations in vitro merely re-establishes the intracellular concentration. In this discussion, we prefer the second alternative, and accordingly, have used the $\mathrm{Co}^{2+}$ activated value. Comparison of this value with the activity theoretically needed shows that the bacteria potentially have a marked excess of enzymatic activity. However, even values for unsupplemented extracts indicate about a threefold excess of peptidase activity over that required for auxotrophic growth. If the level of peptidase activity found in vitro is really present within the organism it is more than sufficient to meet auxotrophic growth requirements, and there is therefore no necessity to induce further peptidases to hydrolyse peptides added to the growth medium. If the high activities in vitro occur in vivo they may in part be required to protect the organism against the build up of inhibitory intracellular concentrations of peptides (Payne, I968; Simmonds, 1970). This calculation is also relevant to the suggestion that the ability of auxotrophic strains to use peptides or amino acids with equal facility makes it doubtful that peptidases normally exist in a latent form within the organism (Simmonds, 1970; Sussman \& Gilvarg, I971), for a few percent of the activity in vitro is sufficient for auxotrophic needs. However, the mutational loss of diglycine peptidase activity can prevent utilization of diglycine by an $E$. coli glycine auxotroph (Kessel \& Lubin, 1963) without apparently affecting growth on glycine; presumably other dipeptidases are sufficient for cellular function but are unable to cleave diglycine at a rate sufficient to meet the high requirement for glycine. On the other hand, we have been unable to isolate dipeptidase deficient mutants of several other auxotrophs, using as selection procedure either inability to utilize dipeptides nutritionally or resistance to dipeptides containing toxic amino acids (J. W. Payne, unpublished results). However, a mutant of $E$. coli deficient in trilysine peptidase activity has been reported (Sussman \& Gilvarg, 1970).

The variability of peptidase activities reported here may be compared with values reported by other workers. Using batch cultures Murayama \& Matheson (I966) reported no significant variation in leucylglycine peptidase activity between exponential-phase and magnesiumstarved organisms. In synchronous cultures of Escherichia coli it has been reported (Kogoma \& Nishi, 1965; Nishi \& Hirose, 1966) that diglycine peptidase activity varies rhythmically 
throughout the growth cycle with maximum activity at the fission period. However, the observed increase in activity was at most about twofold and thus much less than the variability observed here.

The studies here indicate that potassium limitation and anaerobic growth invariably stimulate dipeptidase activity, and that least activity is found with carbon-limited and batchgrown organisms. These general findings can be correlated with other observations that batch-grown exponential phase organisms frequently have lower dipeptidase activity than those from stationary-phase (Sussman \& Gilvarg, 197I). However, such stationary phase organisms are starving for one (or more) essential nutrient and thus are not directly comparable to chemostat cultures which are bacteria growing under nutrient limitation; these nutrient-limited organisms are more analogous to those in the brief stage that separates the exponential phase from the stationary phase in batch-grown cultures. In view of Simmonds' conclusion (Simmonds, 1970), that latency is especially prominent in 'aged' organisms it would be of interest to measure the effects of slow growth rates upon peptidase activity by varying the dilution rate of chemostat cultures.

Simmonds and her co-workers have suggested, mainly from studies of non-growing bacteria exposed to low $\mathrm{pH}$ values for long periods, that acid conditions may produce crypticity or inhibition of peptidases. Although the external $\mathrm{pH}$ may influence the intracellular $\mathrm{pH}$ under certain conditions (Kashket \& Wong, 1969), growth at $\mathrm{pH} 5.5$ does not apparently overcome the homeostatic mechanisms, for we were unable to detect large changes in peptidase activity by altering the $\mathrm{pH}$ of growth.

These findings on peptidase activities may be related to reports on protein turnover. The proteins in growing bacteria (in batch culture) may not turn over appreciably (Podolsky, 1953; Fox \& Brown, I96I; Nishi \& Kogoma, 1965; Willetts, 1967b), although extensive degradation has been reported for organisms under starvation conditions (Mandelstam, I958, I960; Horiuchi, Horiuchi \& Mizuno, I959; Schlessinger \& Ben-Hamida, I966; Willetts, 1967a). However, Nath \& Koch (1970) have recently shown that about 2 to $7 \%$ of the total protein of organisms growing in minimal medium under various nutritional conditions (that may allow growth or cause starvation) is subject to rapid degradation. The calculation in the appendix again indicates that the peptidase activity measured in vitro is more than adequate to account for such levels of protein degradation. It would be interesting to find whether there is any correlation between the extent of protein degradation, and the variation of peptidase activities with nutrient limitation.

\section{APPENDIX}

Valine requirement of organism versus divaline peptidase activity

Let I $\mathrm{mg}$ dry wt of bacteria contain $500 \mu \mathrm{g}$ protein.

Let valine (mol. wt $\mathrm{I}_{7}$ ) be approximately $5 \%$ of total protein.

Amount of valine in protein $=0.05 \times 500 \times \mathrm{I} / \mathrm{II} 7=0.2 \mathrm{I} \mu \mathrm{mol}$.

This amount is required by the culture to double in mass in, say, 60 min generation time.

Specific activity of divaline peptidase required to provide this amount $=0.2 \mathrm{I} \times \mathrm{I} / 0.5 \times$ $\mathrm{I} / 60=0.007 \mu \mathrm{mol} / \mathrm{min} / \mathrm{mg}$ protein. Average specific activity in vitro of divaline peptidase $\left(\mathrm{Co}^{2+}\right.$ activated $)=0.5 \mu \mathrm{mol} / \mathrm{min} / \mathrm{mg}$ protein; this represents about a $70-$ fold excess over that required.

I am indebted to Mr J. R. Hunter for supplying all the chemostat-grown organisms. Thanks are also due to Miss S. Stainer and Mr A. R. Blake for their technical assistance. 


\section{REFERENCES}

Ellwood, D. C. \& Tempest, D. W. (1969). Control of teichoic and teichuronic acid biosynthesis in chemostat cultures of B. subtilis var. niger. Biochemical Journal IIx, I-5.

Evans, C. G. T., Herbert, D. \& Tempest, D. W. (1970). Construction of a chemostat. In Methods in Microbiology, Vol. 2, pp. 277-327. Edited by J. R. Norris \& D. W. Ribbons. London and New York: Academic Press.

Fox, G. \& Brown, J. W. (196I). Protein degradation in Escherichia coli in the logarithmic phase of growth. Biochimica et biophysica acta 46, 387-389.

Hestrin, S., Feingold, D. S. \& Schramm, M. (I955). $\beta$-Galactosidase from Escherichia coli. In Methods in Enzymology, Vol. I, p. 24I. Edited by S. P. Colowick and N. O. Kaplan. London and New York: Academic Press.

Hogness, D. S., CoHn, M. \& Monod, J. (1955). Studies on the induced synthesis of $\beta$-galactosidase in Escherichia coli. Biochimica et biophysica acta 16, 99-1 I6.

Horiuchi, T., Horiuchi, S. \& Mizuno, D. (1959). Degradation of RNA in Escherichia coli in phosphorus deficient culture. Biochimica et biophysica acta 3I, 570-572.

KASHKET, E. R. \& WONG, P. T. S. (1969). The intracellular pH of Escherichia coli. Biochimica et biophysica acta $193,212-214$.

KesseL, D. \& LuBIN, M. (1963). On the distinction between peptidase activity and peptide transport. Biochimica et biophysica acta 7r, 656-663.

$\mathrm{KoCH}$, A. L. \& Levy, H. R. (1955). Protein turnover in growing cultures of Escherichia coli. Journal of Biological Chemistry 217, 947-957.

Kogoma, T. \& Nishi, A. (1965). Rhythmic variations in proteolytic activities during the cell cycle of Escherichia coli. Journal of General and Applied Microbiology II, 32 I-329.

MANDELSTAM, J. (1958). Turnover of protein in growing and non-growing populations of Escherichia coli. Biochemical Journal 69, I I0-I 19.

MANDELSTAM, J. (1960). The intracellular turnover of protein and nucleic acids and its role in biochemical differentiation. Bacteriological Reviews 24, 289-308.

MANDelstam, J. (196I). Induction and repression of $\beta$-galactosidase in non-growing Escherichia coli. Biochemical Journal 79, 489-496.

Matheson, A. T. \& Murayama, T. (1966). The limited release of ribosomal peptidase during formation of Escherichia coli spheroplasts. Canadian Journal of Biochemistry 44, I407-1415.

Matheson, A. T. \& TsaI, C. S. (1965). A ribosomal peptidase from Escherichia coli B. Canadian Journal of Biochemistry 43, 323-329.

Murayama, T. \& Matheson, A. T. (1966). The effect of magnesium starvation on the localization of peptidase activity in Escherichia coli B. Canadian Journal of Biochemistry 44, 654-657.

NAтH, K. \& Косн, A. L. (1970). Protein degradation in Escherichia coli. Journal of Biological Chemistry 245, 2889-2900.

Nishi, A. \& Hirose, S. (1966). Further observations on the rhythmic variation in peptidase activity during the cell cycle of various strains of Escherichia coli. Journal of General and Applied Microbiology 12, 293-297.

Nishi, A. \& Kogoma, T. (1965). Protein turnover in the cell cycle of Escherichia coli. Journal of Bacteriology 9o, 884-890.

PAYNE, J. W. (1968). Oligopeptide transport in Escherichia coli: specificity with respect to side chain and distinction from dipeptide transport. Journal of Biological Chemistry 243, 3395-3403.

PAYNE, J. W. (197I). Utilization of prolyl peptides by Escherichia coli. Biochemical Journal 123, 255-260.

PAYNE, J. W. (1972). The characterization of dipeptidases from Escherichia coli. Journal of General Microbiology 71, 267-279.

Payne, J. W. \& Gilvarg, C. (197I). Peptide transport. Advances in Enzymology 35, 187-244.

PoDolsky, R. J. (1953). Protein degradation in bacteria. Archives of Biochemistry and Biophysics 45, 327-340.

ROTMAN, B. \& SPIEGELMAN, S. (1954). On the origin of the carbon in the induced synthesis $\beta$ galactosidase in Escherichia coli. Journal of Bacteriology 68, 419-429.

Schlessinger, D. \& Ben-Hamida, F. (1966). Turnover of protein in Escherichia coli starving for nitrogen. Biochimica et biophysica acta I19, I7 1 - 182 .

SImmonds, S. (1970). Peptidase activity and peptide metabolism in Escherichia coli KI2. Biochemistry 9, I-9. 
Simmonds, S. \& TOYE, N. O. (1966). Peptidases in spheroplasts of Escherichia coli кI2. Journal of Biological Chemistry 24I, 3852-3860.

Simmonds, S. \& TOYE, N. O. (1967). The role of metal ions in the peptidase activity of Escherichia coli KI 2. Journal of Biological Chemistry 242, 2086-2093.

STONE, D. (1953). Some aspects of the hydrolysis of proline peptides by a prolineless mutant of Escherichia coli. Journal of Biological Chemistry 202, $82 \mathrm{I}-827$.

Sussman, A. J. \& Gilvarg, C. (1969). Protein turnover in amino acid starved strains of Escherichia coli KI2 differing in their ribonucleic acid control. Journal of Biological Chemistry 244, 6304-6306.

Sussman, A. J. \& Gilvarg, C. (197I). Peptide transport and metabolism in bacteria. Annual Reviews of Biochemistry 40, 397-408.

VAn LeNTEn, E. J. \& Simmonds, S. (1967). Dipeptidases in spheroplasts and osmotically shocked cells from Escherichia coli KI2. Journal of Biological Chemistry 242, I439-I 444.

WILLETTS, N. S. (1967a). Intracellular protein breakdown in non-growing cells of Escherichia coli. Biochemical Journal 103, 453-461.

WILLETTS, N. S. (1967b). Intracellular protein breakdown in growing cells of Escherichia coli. Biochemical Journal 103, $462-466$. 